\title{
PROGRESSIVE ADDISONIAN PERNICIOUS ANEMIA, SUCCESSFULLY TREATED WITH INTRAVENOUS CHOLINE CHLORIDE
}

\author{
By F. B. MOOSNICK, E. M. SCHLEICHER,1 AND W. E. PETERSON \\ (From the Department of Internal Medicine, Minneapolis General Hospital, Minneapolis)
}

(Received for publication August 22, 1944)

Sudden failure of an occasional patient afflicted with Addison-Biermer's disease (Addisonian pernicious anemia) to respond to adequate amounts of purified liver extracts in the absence of factors known to interfere with a therapeutic response has been observed by us during the past few years in the Minneapolis General Hospital. The occurrence of a refractory state for no apparent reasons has also been noted by others $(1$ to 4). Because little is known about the chemical nature of the liver factor (anti-pernicious anemia factor) or the physiologic dose, including the development of a progressive anemic state under adequate amounts of purified liver extracts, we felt justified in publishing an observation which we believe may indicate one of the mechanisms responsible for a sudden refractory behavior of certain patients. We could demonstrate in a male afflicted with Addisonian pernicious anemia that fatty liver and fatty metamorphosis of the bone marrow organ were apparently factors in the development of a progressive anemic state.

Without further administration of liver extract, the progressive anemic state was brought into remission by means of the administration of a 5 per cent solution of choline chloride (Merck), 20 ml. intravenously for 16 successive days. The doses were given at a rate of $1 \mathrm{ml}$. per minute. The first dose caused flushing and some nausea after $10 \mathrm{ml}$. were administered. No significant drop in blood pressure was observed. The successive doses were well tolerated.

\section{CASE HISTORY}

The patient, a male, who for several years responded in a characteristic manner to the administration of liver extracts intramuscularly and maintained a normal peripheral blood status on $3 \mathrm{ml}$. monthly of fairly crude liver extracts containing about $1 / 2$ to 2 U.S.P. units per ml., developed a progressive anemic state when purified liver extracts were substituted containing about 10 and 15 U.S.P. units per $\mathrm{ml}$. The peripheral erythrocyte quantity, formerly ranging between 5 and 5.6 mill. per

1 Parke Davis Fellow in Clinical Hematology. c. mm. under crude liver extract therapy, gradually dropped within 3 months to a level ranging between 3.5 and 4.0 mill. per c. $\mathrm{mm}$. on 20 to 45 U.S.P. units per month. The patient then developed an increasing sensitivity to purified liver extracts and all attempts to desensitize him by means of procedures recommended in the literature met with failure $(5,6)$. At this time also, the crude extract, as well as oral liver extract, produced the same allergic symptoms as did the purified liver extracts. The development of a progressive jaundice, several days after the administration of $3 \mathrm{ml}$. of a purified liver extract, given by error, led to his transfer from our dispensary to the hospital for a general work-up.

This 61-year-old white male, who was hospitalized twice before because of classical Addisonian pernicious anemia in severe relapse responding characteristically to purified liver extracts, entered the hospital this time with the complaint of itching, nausea, vomiting, and jaundice for 2 days following the intramuscular injection of 30 U.S.P. units. On the fifth day, he noted dull aching pain in the right upper quadrant. He passed dark brown urine and light colored stools, and the icterus of the skin became progressively deeper. Physical examination revealed a marked hypertension (B.P. 220/150) and a markedly yellow skin and sclera. The liver extending about $6 \mathrm{~cm}$. below the costal margin was smooth with a sharp edge and non-tender. There were no other essential findings. Routine laboratory findings were as follows: hemoglobin 13.5 grams (Newcomer) $=85$ per cent, erythrocytes 3,870,000; color index 1+; white blood cells 5100 ; reticulocytes 0.5 per cent. The peripheral blood showed neutrophiles 46 per cent; lymphocytes 39 per cent; monocytes 11 per cent; eosinophiles 3 per cent; basophiles 1 per cent. A few megalocytes and a small number of poikilocytes were observed. Thrombocytes numbered 140,000 per c. mm. The mean corpuscular diameter (Halometer) was 8.6 microns. Icteric index was 105. The Van den Bergh test, direct and indirect, was positive. Prothrombin time for the patient was 40 seconds, for the control, 24 seconds. Wassermann, Kahn, and Rytz tests were negative. Plasma proteins were: total 8.56; albumin 5.0 ; globulin 3.01 ; and fibrinogen 0.55 grams per cent. Urine showed a specific gravity of 1.021 , albumin $1+$, erythrocytes 4 to 5 , leukocytes 1 to 2 per high-powered field, and numerous cases. Roentgenologic examinations of the gastro-intestinal tract were negative. Lipemia of the plasma could not be demonstrated.

Needle liver biopsy was performed and several specimens from various areas were obtained. The histologic 
diagnosis was acute catarrhal jaundice and moderate severe fatty liver. (Figure 1-B). Simultaneously with the liver biopsy, sternal marrow was aspirated from the proximal end of the body of the sternum at the level of the second interspace and worked up by one of us (E. M. S.), according to methods published elsewhere $(7,8)$. The smear preparations made from the aspirated marrow tissue showed that the marrow organ was moderately severely megaloblastic, indicating that a deficiency of the anti-pernicious anemia factor existed. The gross marrow units (marrow particles) were numerous, varied in size, and ranged between 1 and $4 \mathrm{~mm}$. (normal 0.5 to $1 \mathrm{~mm}$.), suggesting that the marrow organ was
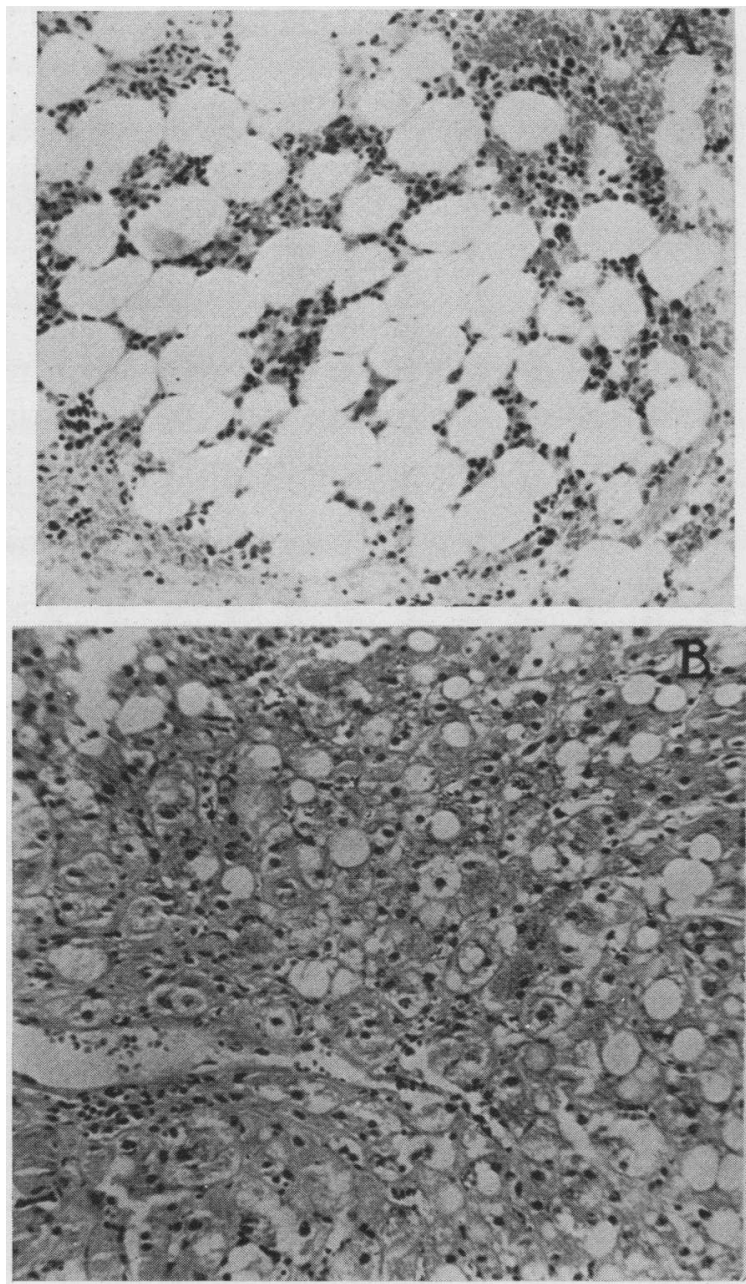

Fig. 1.

A. Average microscopic marrow unit (sternum) before choline therapy showing hypoplasia and severe fatty metamorphosis.

B. Liver (needle biopsy) before choline therapy showing fatty metamorphosis and pattern of catarrhal jaundice. either hypertrophic or the individual units contained an excess amount of fat. The heparinized marrow specimen was transferred into a Wintrobe hematocrit tube and centrifuged at 1500 R.P.M. per minute for 5 minutes. The following layers were obtained from the top of the tube down: fat 10 ; plasma 30 ; nucleated cells or immature myeloid and erythroid cells (M-E) 17; mature erythrocytes derived from ruptured sinusoidal-blood vascular systems (Er.) 48 volumes per cent. ${ }^{2}$ The micro-

2 The normal range of the sternal volume percentage of the adult male as determined by one of us (E. M. S.) is believed to be : fat 0.5 to 3.0 ; plasma 39 to 48.5 ; $\mathrm{M}-\mathrm{E}$ 4 to $6 ;$ Er. 45 to 54 .
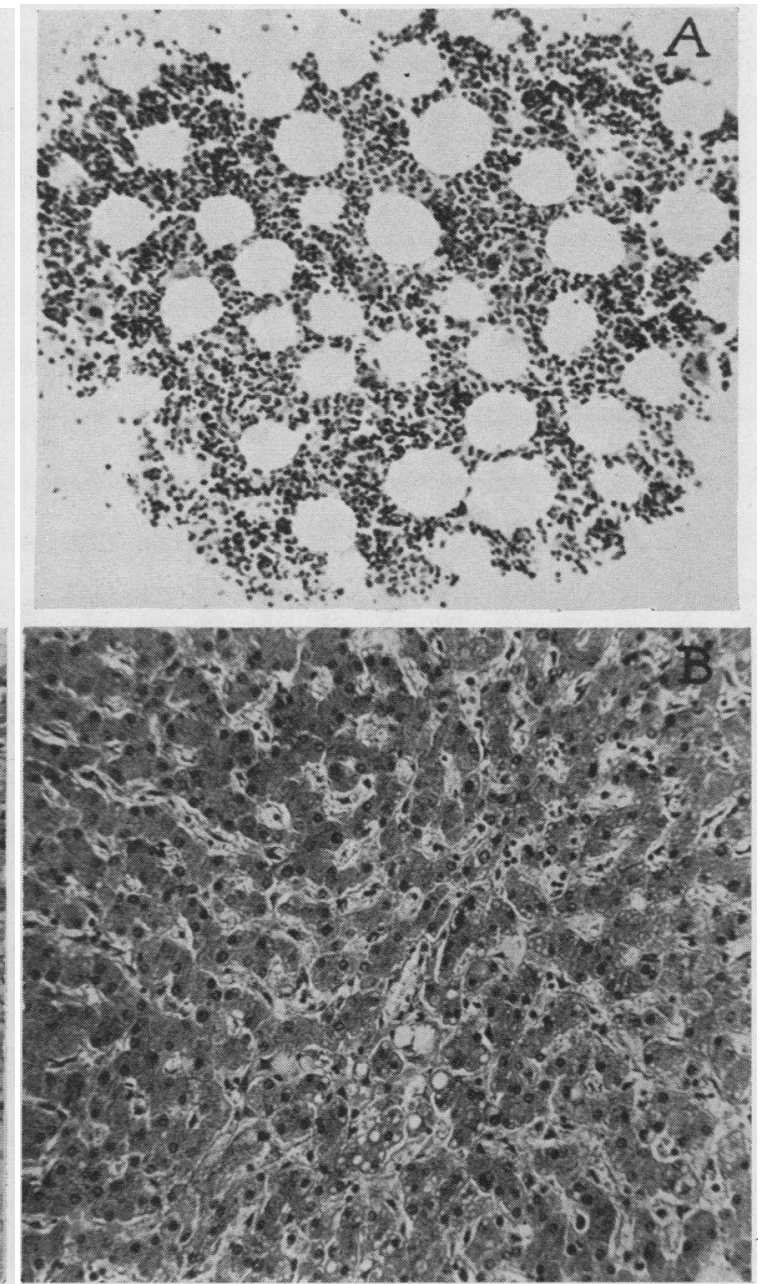

FIG. 2

A. Average microscopic marrow unit (sternum) after choline therapy showing hyperplasia and normal maxinum number of fat spaces.

B. Liver (post-mortem) showing some fat cells and polymorphonuclear neutrophils. 
scopic marrow units (bounded by 25 squares of the Whipple ocular micrometer when the following optical system is used, eyepiece $10 \times$ and objective $10 \times$, showed conspicuous fatty metamorphosis and a moderately severe hypoplasia of the hematopoietic tissue. The number of fat spaces ranged between 45 and 60 per 25 squares. $^{3}$

Figure 1-A shows an average microscopic unit with fatty metamorphosis and hypoplasia. Generally the microscopic marrow units in uncomplicated cases are hyperplastic and show none to a few fat spaces. On the basis of the fatty liver and marked increase of the fat in the marrow organ, both macroscopic and microscopic, one of us (E. M. S.) advanced the hypothesis that the fatty state was the factor responsible for the development of the progressive anemic state in our patient and that the fatty state may have resulted from a choline insufficiency. The choice of choline and dosage for reducing the fat deposit in the liver and bone marrow organ were influenced by several factors. First, by the observations of others ( 9 to 14) that choline is a lipotropic substance important in the mechanism of fat transportation and deposition. Second, on the data accumulated by one of us (E. M. S.) on the lipotropic effect of daily intravenous injection of $10 \mathrm{ml}$. of a 1 per cent choline chloride solution (Merck) over a period of from 5 to 10 days on the fat volume percentage of the sternal part of the adult human bone marrow organ of cases with cirrhosis of the liver (confirmed by needle biopsy) and carcinoma of the stomach, with and without metastases to the bone marrow. These data showed that a 1 per cent solution reduced excess marrow fat to a normal volume percentage within a few days without causing alarming symptoms. No appreciable changes of the peripheral blood status were observed. And third, the daily dosages were determined on the basis of work done by one of us (F. B. M.) relating to the effect of choline chloride on the blood pressure of patients with essential hypertension.

Figure 3 shows the peripheral blood status before, during, and after choline chloride therapy and the fat volume percentage of the bone marrow organ before and at the time therapy was discontinued. As can be easily seen, there was no appreciable change of the peripheral blood status during a 12-day pre-treatment period. Administration of choline chloride (Merck) was followed by an increase of reticulocytes reaching a peak of 5.5 per cent on the third day. The obtained reticulocyte peak is generally expected for a red cell quantity about 3.0 mill. per c.mm. Simultaneously, the anemic state improved and the icteric index began to decrease. On the sixteenth day, the peripheral blood status was well within the normal range and choline therapy was discontinued. The icteric-index at this time was 26 units. Marrow

${ }^{3}$ If the recommended optical combination setup cannot be made, one may determine the normal microscopic marrow unit with any eyepiece and low power object available. Serial sections of an average normal gross marrow unit are moved under the Whipple micrometer and the number of squares noted that form the boundary of the maximum section. was aspirated from the distal end of the body of the sternum at the level of the second interspace. The obtained specimen was worked up in the same manner as the first specimen. The gross marrow units were in size and number within the normal range. The hematocrit showed the various layers within the normal limits: fat 1 ; plasma 41 ; M-E 6 ; Er. 52 volumes per cent. The microscopic marrow units showd a remarkable adjustment toward normality and the fat spaces ranged between 20 to 35 per 25 Whipple ocular micrometer squares (Fig. 2-A).

We were not able to get simultaneous information on the status of the liver when choline therapy was discontinued because the patient refused to submit to another liver biopsy. We realize that the lack of this information weakens any conclusions we wish to draw with respect to the effect of choline on the liver fat; we nevertheless believe that a favorable action on the fat deposit had occurred because from the histologic point of view the liver was practically normal when the patient expired some months later from pneumonia (Figure 2-B).

\section{DISCUSSION}

For as yet unknown reasons, man may develop a spontaneous choline deficiency resulting in the production of a fatty liver and bone marrow organ. In certain individuals afflicted with Addison-Biermer's disease (Addisonian pernicious anemia), a progressive anemic state may develop as a sequel of the fatty metamorphosis. The latter state may well be enhanced by purified liver extracts because they contain no choline or amounts too small to be effective with respect to fat transportation and deposition. One author believes that the body stores of choline cannot be utilized to prevent development of a choline deficiency. This is of interest because our patient consumed 3 balanced meals a day and seemingly failed to utilize choline considered (13) to be a structural constituent of foods.

While we had no direct evidence of choline deficiency, it appeared to be most likely that the substance was deficient because of the conspicuous fatty liver and fatty bone marrow organ. The dramatic response of the anemic state and adjustment of the mean corpuscular diameter of the erythrocytes to choline therapy without supplementary antianemic substances would indicate that an adequate amount of hematopoietin was stored, although not elaborated by the fatty liver in sufficient amounts to prevent a deficiency state of the substance. Further, it is likely that fat deposition above a certain volume percentage in 


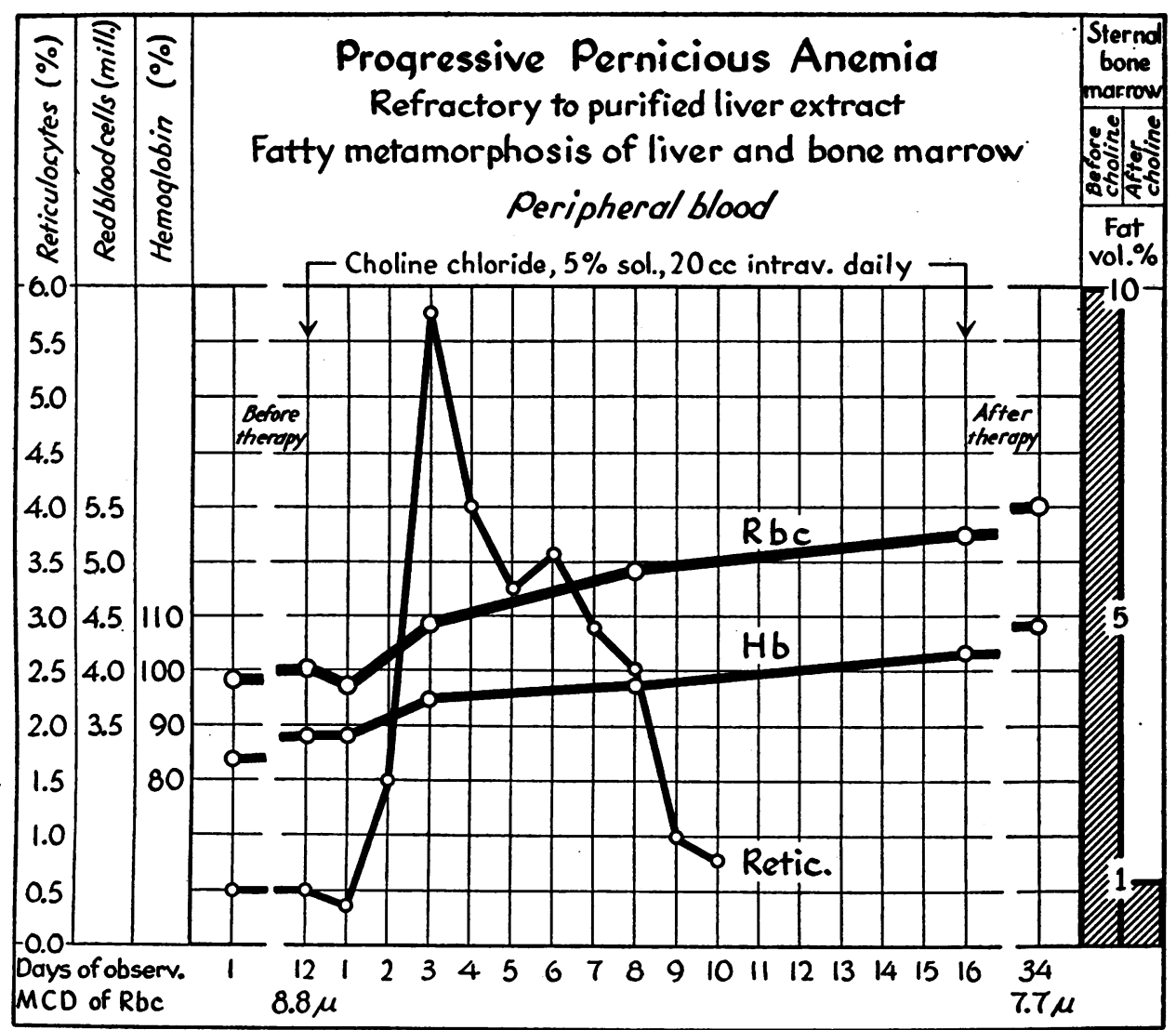

Fig. 3. Peripheral Blood Status Before, During, and After Intravenous Choline Chloride (Merck) and Volume Percentage of Sternal Marrow Fat, Before and After Choline Therapy

the marrow organ may cause a disturbance in the utilization of the hematogenic substance should some be elaborated by the liver. While we were able to obtain data on the status of the bone marrow organ before and after choline therapy and on the liver before the administration of the lipotropic factor, we failed to get the cooperation of the patient for another liver biopsy when choline therapy was discontinued. As already mentioned elsewhere in this paper, the liver was found macroscopically and microscopically normal when the patient expired some months later from pneumonia. With the exception of the first dose, no ill effects nor any change of the blood pressure was observed during the administration of choline over the treatment period. Because of our inability to desensitize the patient to liver extracts, readministration of crude liver extracts could not be carried out. He was placed on Ventriculin after the thirty-fourth day of observation. We could show in a clear-cut manner that an adequate amount of hematopoietin was stored in the liver and that the administration of choline in some way expedited the elaboration of the substance, bringing about a remission of the anemic state. The response to choline was in every respect compatible with that induced by potent liver extracts. Whether this type of therapy is effective in cases other than those afflicted with Addison-Biermer's disease (Addisonian pernicious anemia) must await further investigation. We see no reason why choline should not be supplemented when purified liver extracts are used, especially when a progressive anemic state develops and fatty metamorphosis of the liver and bone.marrow organ appears to be the complicating factor. The remission of the anemic state on choline therapy is not in accord with the observation of one 
worker (15) who gave choline hydrochloride to dogs made polycythemic and noted a depressing action of the substance on the erythropoiesis. It is worthwhile to note that others (1) employed blood transfusions to overcome the refractory anemic state.

\section{SUM MARY}

1. Report is made of a case afflicted with Addison-Biermer's disease (Addisonian pernicious anemia) who developed a progressive anemic state under adequate amounts of purified liver extracts.

2. The anemic state responded well to choline chloride (Merck), 5 per cent solution, $20 \mathrm{ml}$. intravenously for 16 days.

3. Biopsied liver and bone marrow tissue showed marked fatty metamorphosis of both organs. This fatty state was believed to have been the reason for the failure of the patient to maintain a normal blood status.

4. The fact that a remission of the anemic state, reduction of the mean corpuscular diameter of the erythrocytes, and return of the bone marrow organ toward a normal histology and volumetry occurred on choline therapy only seems to indicate that an adequate amount of hematopoietin (antipernicious anemia factor) was stored in the liver but not effectively elaborated because of the fatty state.

5. It is assumed that purified liver extracts contain either no choline or amounts too small to be effective with respect to fat transportation and deposition in certain individuals.

6. The amount of choline and the number of days of administration essential for the reestablishment of a physiologic state of the liver and bone marrow organ and elaboration of the hematopoietin in man await further investigation.

7. It is suggested that choline chloride (Merck) or substances containing choline may be supplemented when fatty metamorphosis of liver or bone marrow organ or both are present, particularly when a progressive anemia develops under ade- quate purified liver extract therapy in the absence of factors known to interfere with a therapeutic response.

We wish to thank Dr. G. E. Fahr, Head of the Department of Internal Medicine, for his interest in the problem and for the permission to publish this case.

\section{BIBLIOGRAPHY}

1. Davidson, L. S. P., Davis, L. J., and Innes, J., Studies in refractory anaemia. Edinburgh M. J., 1943, 50, 431.

2. Mulholland, H. B., Intravenous liver extract in the therapy of pernicious anemia. Ann. Int. Med., 1937, 11, 671.

3. Sharp, E. A., Directing head of the Anemia Laboratory, Harper Hospital, Detroit, Mich. Personal communications to the authors.

4. Sommers, B., Directing head of the Anemia Clinic, Minneapolis General Hospital. Personal communications to the authors.

5. Feinberg S. M. Alt, H. L., and Young, R. H., Allergy to injectable liver extracts; clinical and immunological observations. Ann. Int. Med., 1943, $18,311$.

6. Kaufman, R. E., Farmer, L., and Reich, C., Allergic reactions to liver extract. Ann. Int. Med., 1943, 19, 768.

7. Schleicher, E. M., Staining aspirated human bone marrow with domestic Wright stain. Stain Technol., 1942, 17, 161.

8. Schleicher, E. M., The volumetric pattern of aspirated normal human sternal marrow of males 18 to 40 years. Am J.. Clin. Path., 1944, 14, 370.

9. MacLean, D. L., and Best, C. H., Choline and liver fat. Brit. J. Exper. Path., 1934, 15, 193.

10. Best, C. H., and Ridout, J. H., Choline as a dietary factor. Ann. Rev. Biochem., 1939, 8, 349.

11. Best, C. H., and Channon, H. J., The action of choline and other substances in the prevention and cure of fatty livers. Biochem. J., 1935, 29, 2651.

12. McHenry, E. W., Choline, the B Vitamins and fat metabolism. Biological Symposia, 1941, 5, 177.

13. McHenry, E. W., and Patterson, J. M., Lipotropic factors. Physiol. Rev., 1944, 24, 128.

14. Okey, R., and Greaves, V. D., Anemia caused by feeding cholesterol to guinea pigs. J. Biol. Chem., 1939, 129, 111.

15. Davis, J. E., Depression of experimental polycythemias by choline hydrochloride or liver administration. Am. J. Physiol., 1939, 127, 322. 